\title{
Actualities on diagnosis, targeting and treating bone metastases in breast cancer with radiotherapy and new drugs
}

Authors:

Fabio Marazzi1, Armando Orlandi2, Stefania Manfrida1, Valeria Masiello1, Alba di Leone3, Mariangela Massaccesi1, Francesca Moschella3, Gianluca Franceschini3-4, Emilio Bria2-4, Maria Antonietta Gambacorta1-4, Riccardo Masetti3-4, Giampaolo Tortora24, Vincenzo Valentini1-4 Affiliations:

1 Fondazione Policlinico Universitario "A. Gemelli" IRCCS, UOC di Radioterapia Oncologica, Dipartimento di Diagnostica per Immagini, Radioterapia Oncologica ed Ematologia, Roma, Italia

2 Fondazione Policlinico Universitario "A. Gemelli" IRCCS, UOC di Oncologia Medica, Dipartimento di Scienze Mediche e Chirurgiche, Roma, Italia

3 Fondazione Policlinico Universitario "A. Gemelli" IRCCS, UOC di Chirurgia Senologica, Dipartimento di Scienze della Salute della Donna e del Bambino e di Sanità Pubblica, Roma, Italia

4 Università Cattolica del Sacro Cuore, Istituto di Radiologia, Roma, Italia

Corresponding Author: Valeria Masiello, valeria.masiello@guest.policlinicogemelli.it (ORCID 0000-0001-7589-7623)

Abstract

The standard of care for metastatic breast cancer $(\mathrm{MBC})$ is systemic therapies with imbrication of focal treatment in case of symptomatology onset. Recently, thanks to implementation of radiological and metabolic exams and development of new target therapies, oligometastatic and oligoprogressive disease presentations are even more common, leading to a change of paradigm of focal treatments. In fact, acknowledgement of behaviour of disease in these setting of patients is carrying aim of radiotherapy towards modalities with radical intent. The aim of this literature review is to analyse available clinical data regarding disease behaviour, imaging, radiotherapy and chemo-radiotherapy integration outcomes for understanding bone metastasis from breast cancer and the potential impact of targeting it.

Keywords: bone metastasis - breast cancer - radiotherapy - diagnostic imaging - target therapy 


\section{Introduction}

In the last years, metastatic breast cancer $(\mathrm{MBC})$ has shown an improvement of outcomes thanks to treatment implementations[1], but prognosis is still critical[2] with a $27 \%$ of reports 5 -year survival rates[3]. Incidence of MBC interests $25-28 \%$ of de novo metastatic, while the rate of metastatic recurrence is reported in $20-30 \%$ of pts in western country and can be even more in low-medium income countries[4]. Over time, for patients the risk of becoming metastatic arises and data describes a cumulative risk of $4.8 \%(4.7-4.8)$ at one year, 5.6\% (5.5-5.6) at two years, 6.9\% (6.8-7.0) at five years, and 8.4\% (8.3-8.5) at ten years[5].

Bone metastasis occur commonly in solid tumors and in $36 \%$ of incidence is from breast cancer [5], with a stratification according to subtypes that shows a tendency of incidence in Luminal subtypes[6]. In a Surveillance Epidemiology End Result (SEER) database retrospective analysis on subtype and incidence of distant metastasis, data on first site of relapse showed that bone metastases commonly interest luminal subtypes (ER+/HER2- $58.52 \%$ and in ER+/HER2+ subtype $47.28 \%$ of incidence), while ER-/HER2 subtype has a high proportion of liver metastases (31.72\%) and triple negative (TN) subtype is more interested by lung metastases (32.09\%), with an incidence of bone metastases respectively of $34.49 \%$ and $36.39 \%[6]$. In another retrospective study by Molnar IA et al., Luminal A subtype presented a tendency of isolated bone metastases presentation in 59\% of cases[7]. In breast cancer, bone metastasis can occur in de novo or recurrent, plurior oligometastatic situation and associated or not with other site of involvement, with a spectrum of prognosis that can differ a lot[6], [8], [9]. According to time, disease presentation and prognosis, metastatic breast cancer patients with bone metastasis can be divided, in the sequent subgroups:

- De-novo plurimetastatic bone-only breast cancer: breast cancer with more than 5 bone metastases at diagnosis

- De-novo plurimetastatic bone-parenchimal breast cancer: breast cancer with more than 5 bone metastases at diagnosis

- De-novo oligometastatic bone-only breast cancer: breast cancer with less than 5 bone metastases at diagnosis 
- De-novo oligometastatic bone-parenchimal breast cancer: breast cancer with less than 5 bone metastases at diagnosis

- Recurrent plurimetastatic bone-only breast cancer: breast cancer with more than 5 bone metastases at recurrence

- Recurrent plurimetastatic bone-parenchimal breast cancer: breast cancer with more than 5 bone metastases at recurrence

- $\quad$ Recurrent oligometastatic bone-only breast cancer: breast cancer with less than 5 bone metastases at recurrence

- Recurrent oligometastatic bone-parenchimal breast cancer: breast cancer with less than 5 bone metastases at recurrence

Etiopathology of bone metastasis is based on multicellular unit (osteoblasts, osteoclasts, bone lining cells, osteocytes) disruption with release of growing factors (TGF-B, FGF, PDGF, IGF) that promote increase of tumor cell growth and further disruption of bone architecture [5], [10]. In particular, molecular hypothesis is that in sclerotic lesion tumor produces growth factors and derived that induce osteoblasts differentiation and inhibit bone resorption, while in lytic lesion tumor-derived production enhance pro-osteoclastogenic differentiation and activity with consequently bone resorption[11].

Bone metastases are a common cause of cancer pain and its possible local mechanisms of aetiology are release of chemical mediators, increased pressure in the bone, microfractures, stretching of the periosteum, reactive muscle spasm, nerve root infiltration, compression of the nerve due to collapse of the bone[12].

Skeletal-related events (SRE) are complications of bone metastasis growing, such as pathological fracture, spinal cord compression, necessity of radiotherapy for pain/impending fracture or surgery to bone, that can compromise patients performance status with reduction of quality of life and limitation to access systemic therapies with a prejudice of survival outcomes[13]. On the other side, thanks to new emerging diagnostic imaging and new drugs[14], we are assisting even more to oligometastatic presentation (de novo or inducted) 
spread[8]. Early detection of metastases and approaches with targeting agents can enhance chance of disease control and implement number of long responders [2], [15], [16].

Associated with systemic therapeutic options, radiotherapy is a possibility for local treatment of bone metastasis both with palliative and eradication intent[17], [18]. Radiobiological aim of radiotherapy is to cause an interruption of this the vicious cycle and cause not only a reduction in osteolytic bone lesions, but also decrease local tumour burden in more radiosensitive tumor subtypes[19]. It is clinically proven that patients present an immediate relief of symptoms in 2-4 weeks[11], [20], [21] and radiologically demonstrate that for intent-to-eradicate treatments, local control at 1- and 2-years reported can arrives to 90.3 and $82.4 \%$ with excellent safety[22].

For this reason, oligometastatic/oligoprogressive patients are even more challenging because physicians can imbricate local treatments such as radiotherapy with new systemic drugs in order to obtain higher progression free survival and in general overall survival benefit. In this setting of patients, radiotherapy also presents a possibility of eradication of sub-clones resistant to systemic therapy.

Here we propose a review of new diagnostic imaging for early detection of bone metastasis in breast cancer, their use for radiotherapy targeting, radiotherapy possibilities in terms of dose and volumes and chemoradiotherapy integration to improve clinical outcomes. Final purpose is to offer an instrument for multidisciplinary management of patients with $\mathrm{MBC}$ and bone metastases presentation.

\section{Diagnostic Imaging for bone metastasis from breast cancer}

Metastatic spread from a primary breast tumor can occur at an early, pre-symptomatic stage, and disseminated cells can lie dormant for years before becoming clinically evident[23]. In some studies [24] [25], it is provided that, into the metastatic process of breast tumours, disseminated cancer cells at early stages of tumour evolution successfully establish themselves in the bone marrow [23]. Based on this theory, 
adjuvant systemic therapy (chemotherapy, target therapy and/or hormone therapy), is always administered, when it is indicated.

A challenge for physicians is to precociously identify bone metastasis in patients during staging and follow up, for defining oligometastatic presentation and select patients with good prognosis for focal non palliative radiotherapy. At the present day, with innovation in morphological and functional exams, novel technologies offer possibility to detect early bone metastases. Even more diagnostic and functional imaging are moving towards this goal. Based on metastasis behaviour, (lytic, sclerotic or mixed) metastases can present different pattern at imaging.

Morphological Imaging. Morphological exams, including radiographs or computed tomography (CT), are related to changes in bone density. To be detected at CT exams, bone metastases need to be at least $1 \mathrm{~cm}$ with a loss of density around $25-50 \%$. Usually breast cancer bone metastasis are lytic, but during treatments, due to response and osteoblastic reaction, they can become peripherally osteosclerotic. CT also allows to define soft-tissue invasion outside bone. Moreover, morphological exams are fundamental to define critical site of bone metastasis which are at risk for SRE.

Conventional MRI sequences with T1, T2 and DWI studies, allow to detect breast cancer bone metastases with a sensitivity reported since to $100 \%[26]$, so they are used in case of doubt of for early detection. MRI allow to visualize lesion in a very precise way, and it is also useful to study integrity of spinal cord and eventually condition of its compression. MRI for only bone study can be performed without contrast, but in case of study of spinal cord or soft-tissue, contrast is required. Recently, also whole-body MRI (WB-MRI) has been developed as possibility of study entire bone compartment, but its utility for clinical practice is still under investigation, especially for early detection of bone metastasis[27]. In literature, data on WB-MRI also provide a quantitative measure of treatment response in skeletal metastases[28].

Functional Imaging. Functional Imaging finds a role in staging and, during follow up, detecting bone metastasis in breast cancer. Bone scintigraphy with $99 \mathrm{mTc}$-radiolabeled diphosphonates is in use since 1970ies, but its sensitivity and specificity is limited respect SPECT/CT and 18 FDG PET/CT from which 
information about treatment response and prognosis are also available[29]. Bone scintigraphy usually detect bone turnover, so metastasis with a prevalent lytic behaviour can be considered as false negative.

In NCCN Breast Cancer Guidelines, for stage since to IIIA (since to T3N1) bone scan is indicated in clinical practice if localized bone pain of in case of elevate alkaline phosphatase, while 18 FDG PET/CT is considered optional[30]. 18 FDG PET/CT is instead considered useful in case of locally advanced or metastatic disease for staging, evaluate treatment response and prognosis[30].

In a recent review by Cook J et al. [28], it is reported that molecular and hybrid imaging, has an increasing role in detecting bone metastases early in their evolution and in monitoring treatment response at early time points. In this sense, functional imaging as emission computed tomography (SPECT/CT), positron emission tomography /CT (PET/CT) or PET/MRI in breast cancer could find a role in identified early patients not responder to systemic therapies for shifting to further line of treatment with a benefit on disease control and cost/effectiveness of health systems.

Diagnostic Imaging for Treatment Planning of Radiotherapy. Morphological Imaging is useful for identify bone lesions and soft tissue invasion. In palliative radiotherapy treatments of bulky metastases, CT scan simulation allow radiotherapist contouring also of soft tissue surrounding. In some cases, co-registration with diagnostic CT scan with contrast can be helpful for distinguish healthy soft tissue from that interested by spread of disease outside bone metastases. MRI is useful for treatments with eradication intent because it allows to be very precise in Gross Tumor Volume (GTV) contouring and spinal cord definition for sparing it. MRI is usually required for Stereotactic Body Radiotherapy (SBRT), in which target of the treatment is the lesion with a millimetric margin and dose are high. Functional imaging is less strictly used for contouring of bone metastasis in breast cancer and hold a function of supporting detecting of lesion at co-registration.

Biopsy on bone metastasis: when imaging is not enough. Metastatic presentation usually required a biopsy for prognostic factors study to confirm nature of disease and indication to systemic therapies. More often, in case of de-novo metastatic patients, soft-tissue or primary tumor undergo pathological study, while in case of relapse, especially for isolated bone presentation, a biopsy of lesion can become mandatory to study 
disease presentation. Other conditions in which biopsy can be mandatory are necessities of differential diagnosis. The differential diagnosis for bone metastases includes chondrosarcoma, primary malignant lymphoma of the bone, multiple myeloma, post-radiation sarcoma, and osteomyelitis. A distinction between acute osteoporotic fractures versus metastatic fractures should be made on radiographic imaging. In osteoporosis, the cortical bone may be preserved; however, cortical bone destruction is typical with metastatic cancer. Another possible cause of bone lesion in differential diagnosis is sarcoidosis because osseous sarcoidosis lesions cannot be reliably distinguished from metastatic lesions on routine MRI studies[31]. 18F-FDG PET/CT is highly sensitive in detecting granulomatous bone marrow infiltration, but an increased 18F-FDG uptake can mimic metastatic disease, reducing the specificity of 18FDG PET/CT when both sarcoidosis and a tumour which may develop bone metastases occur in the same patient[32].

\section{Radiotherapy treatments options and new drugs}

Radiotherapy effect on bone metastasis. In-human data of pathological damage by radiotherapy on bone metastases are few. Tissue damage by radiotherapy is mediated by sub-lethal damage of free radical generated by free-radical or, in case of high doses, also direct damage on DNA[33]. In fact, higher doses for fraction, as in stereotactic radiotherapy (SBRT), can promote direct cytotoxic, endothelial disruption with vascular death [34], [35] (Figure 1). Final effect of this damage is reduction of pain (for stopping of biomolecular pain modulation mechanisms), decrease of osteolysis and tumor burden[36]. Radiotherapy with palliative intent causes an interruption on neuromodulatory algic mechanism by early depletion of inflammatory cells, thanks to inhibition of the inflammatory cells[12]. Main trigger of pain modulation by bone metastases are nerve growth factor (NGF), bradykinin, serotonin, adenosine tri-phosphate, $\mathrm{H}+$, lipids (prostaglandin E2) and degenerin family of ion channels[12].

Decrease of osteolysis is mediated by osteoclasts apoptosis, as in vitro data showed[37]. Radiotherapy can also promote re-ossification process from 3-6 weeks from the end of radiotherapy and reaches highest 
degree within 6 months[11]; ossification process is realized in $65 \%$ to $85 \%$ of lytic metastases in unfractured bone[12].

In a study by Steverink et al.[38] on ten biopsy of vertebral metastasis who underwent a single pre-operative SBRT of 18 Gy on spinal bone lesion a change of tissue in 21 hour in sense of necrosis in $83 \%$ of sample with a consistent reduction of mitotic activity and vessel density (especially in renal cell metastases who are enriched of vessels). Biopsy also showed a persistence of T-cell and natural kill cell density after SBRT, probably immune-related reactions against antigens exposed by tumor cell damage starts in a further phase.

From radiobiological data on primary tumor, in which lesions since to $4 \mathrm{~cm}$ were treated with definitive radiotherapy, 3-y local control of 81 and $100 \%$ were respectively achieved with doses of 70-80 Gy and $>80$ Gy [39]. Some authors hypotizes that a large single fraction could be more advantageous on breast cancer, compared with prolonged fractionated radiotherapy[40]. For the tissue damage caused, radiotherapy can be considered crucial as local ablative treatment in oligometastatic breast cancer setting especially when a BED>75Gy [41].

Dose and volumes of Radiotherapy treatments. Indication of dose and volumes of radiotherapy depends by intention of treatment. In palliative setting, radiotherapy aims to control symptoms and local growing of disease. It is usually associated to drugs for pain control and orthopaedic multidisciplinary evaluation. Volumes of radiotherapy in palliative setting, usually includes all the bone compartment which contains the lesion, with margins. Historically, these treatments are administered with 3D conformal treatment plan with one or more fields of therapy, according to achieving organ at risk sparing, but at the present day, especially in case of re-treatment, even more sophisticated techniques such as Intensity Modulated Radiotherapy (IMRT) or Volumetric Modulated Arch Therapy (VMAT) can be chose for optimizing dose distribution, avoiding missing target and preserving organ at risk, especially spinal cord. Palliative radiotherapy is brief with administration of 8-20 Gy in 1-5 Fr, in order to reach a pain relief or neurological symptoms degeneration control in some weeks [20], [21] (Table 1a). In a metanalysis of Chow E et al. it is reported that efficacy of single-fraction RT and multi-fractions (since to $30 \mathrm{~Gy}$ ) are equivalent in terms of pain control, but rate of re- 
treatment are 2.5-fold higher in single-fraction arms[42]. Patients who underwent surgery for SRE can benefit of radiotherapy. A prospective study on bone metastases with spinal cord compression showed that responsiveness of breast cancer tumor (that was classified intermediate) is linked to schedule of 30 Gy given with 10 fractions and dose escalation is not related to an improvement of outcomes[43].

Treatments with intent of eradication in oligometastatic setting are usually given in few days but, in this case, total doses reach a higher biological equivalent dose (BED), of at least 75 Gy[41], [44], [45] (Table 1b). In this setting of treatments, usually a more sophisticated techniques are used to conform volumes, and stereotactic body radiotherapy technique (SBRT) is often applicated for sparing organ at risk and give higher doses on the core of GTV. SBRT requires strictly system of immobilization and co-registration with MRI is mandatory to detect bone lesion and for spinal cord identification[46].

In literature are reported few retrospective and prospective series about oligometastatic breast cancer, but evidences reported show that a treatment direct to metastases (surgery or radiotherapy) is significantly related to survival outcomes at 10-20 years[17], [18]. Patients who are candidate to this kind of treatments need to be carefully selected in terms of prognosis[47]. In general, breast cancer is a favourable prognostic factor for OS in oligometastatic patients who underwent SBRT (HR, 0.12; 95\% Cl, 0.07-0.37) [48]. Another prognostic factors that has been found related to OS in a retrospective SBRT for oligometastatic analysis was BED>75Gy[44]. In a study by Milano MT et al. survival outcomes of SBRT in 48 oligometastatic breast cancer treated for extracranial metastases showed that bone-only oligometastatic present a younger age, usually are hormone-responders and synchronous with diagnosis [17]. In this study, OS and freedom-fromwidespread metastases (FFWM) were better in bone-only group (12 pts) and patients underwent a radiotherapy with a median EQD2 of $57.3 \mathrm{~Gy}$ [38.3-70]. In a phase II prospective trials, oligometastatic breast cancer patients were treated on all sites of metastases with SBRT (30-45 Gy in $3 \mathrm{Fr}$ ) or IMRT (60 Gy in $25 \mathrm{Fr}$ ). Results showed thar 60 on 92 metastatic lesions were in the bone and $80 \%$ of pts included were Luminal A. In this study, 1- and 2-year PFS was 75\% and 53\%, respectively; two-year LC and OS were $97 \%$ and $95 \%$, respectively, while only 1 bone lesion treated on 60 relapsed in spine (but was treated with $17 \mathrm{~Gy}$ in $3 \mathrm{Fr}$ )[49]. 
In another study of 2015 by Yoo GS et al. 50 patients with bone metastases who underwent radiotherapy for a median dose of 30 Gy (20-60 Gy) were retrospectively studied. The analysis of Yoo GS showed that patients treated with a BED of at least 50 Gy presented better 5-y LC and OS[50]. In a prospective cohort of 50 pts with breast cancer, 68 spine bone metastasis were treated with a single fraction radiosurgery for a total mean dose of 19 Gy (15-22.5 Gy) with a $96 \%$ of pain control and local control at 15 months was $100 \%[40]$. In a mixed cohort of 22 oligometastatic and oligoprogressive patients, $32 \%$ were affected from breast cancer and were treated with dose to most spinal and non-spinal metastases is 35 and $50 \mathrm{~Gy}$, respectively, in five fractions[51]. Local control achieved was 91\% at 1-y 1-year LC 91.2\%), while median PFS and OS are 10.1 and 37.3 months, while PFS of OP and OM group is 6.6 and 10.6 months, respectively.

Some patients are not candidate to Stereotactic RadioSurgery (SRS), for presence of more than 3 lesions or for proximity to spinal canal, and an intermediate solution to achieve a better local control is to administer a simultaneous integrated boost (SIB) on GTV, treating whole vertebra with palliative dose and fractionation. In a cohort of 12 patients, of which only was affected by breast angiosarcoma (with a different radiosensitive respect breast carcinoma), treated with a SIB of 40 Gy and 30 Gy on whole vertebra given in $10 \mathrm{Fr}$, a 1-y LC was $93 \%[52]$.

At the present time, there is a great inhomogeneity in dose prescription especially for extraspinal bone metastasis and the need of consensus guidelines supported by evidences is necessary[53], [54].

Cytotoxic chemotherapy and radiotherapy. Systemic therapy is still the fundamental treatment for all molecular subtypes in the management of bone metastases of $\mathrm{MBC}[30]$, [55]. Drug's choice is influenced by immunophenotype, previous treatment and tumor spread[30], [55]. In TNBC or hormonoresistance MBC anthracycline- or taxane-based regimens are preferred treatment[56], [57]. Recently, therapeutic options after anthracycline- and taxane-resistant disease were increased. In fact, some cytotoxic drugs after first line chemotherapy treatment are become available. In the last years, the monotherapies eribulin[58] and nanoparticle albumin-bound paclitaxel[59] have been added to the treatments that have long been available 
as capecitabine, vinorelbine, cyclophosphamide, gemcitabine and pegylated liposomal doxorubicin[60], [61]. Also, combination therapies such as paclitaxel plus gemcitabine or carboplatin plus gemcitabine could represent an alternative option, but sequential monotherapy is usually preferable in MBC setting[58], [59]. Generally, bone metastases had the low response rates to chemotherapy. For this reason and for the need for a rapid improvement of pain symptoms, these systemic treatments are often imbricated with palliative radiant treatment. At the same time, in the condition of oligometastatic, it may be necessary to imbricate radiant treatments with cytotoxic treatment with a radical intent. In both cases, considering the significant risk of myelosuppression of both treatments, radiotherapy is almost never concomitant with systemic treatment. The clinicians must merge these treatments in order to avoid the overlap of the specific nadirs of bone marrow toxicity. The sequence of these treatments is dictated by the need to prioritize a systemic control of disease versus a locoregional control (oligoprogressive) or the pain control.

Hormonal therapy and radiotherapy. In MBC patients, bone metastases more often derived from HR-positive tumors as previously described[7]. In this case hormonal therapy (ET) is the preferred choose in most cases, except for rapidly progressive disease or in case of visceral crisis, where cytotoxic drugs remain the preferred option[55]. In recent years, the introduction of everolimus (M-TOR inhibitor)[62] and alpelisib (PI3KCA inhibitor)[63] in hormone refractory disease and CDK4/6 inhibitors[14], [64] in both hormone-sensitive and hormone-refractory disease has made hormonal sequence more complex and often longer.

The spread in clinical practice of these treatments, in a metastatic setting characterized by the frequent presence of bone metastases, has also determined the need to imbricate these systemic therapies with radiant treatment with a palliative or radical purpose. If the hormonal treatment alone, characterized by an excellent toxicity profile, not arise problem for a combination treatment (radiotherapy with hormonal therapy), the introduction of target therapy associated with hormone therapy instead raises the problem of timing of these effective treatments. No prospective study was addressed to establish the best combination schedule between hormone-associated target therapy and palliative or radical radiotherapy. The presence of continuous and semi-continuous therapeutic schedules for these target therapy, implying the possible need to discontinue treatment in case of necessity to decrease cumulative toxicity. As regards everolimus 
and alpelisb, in the absence of clinical data, no biological contraindications can be postulated at the basis of the need for drug's suspension during radiant treatment. Vice versa, using CDK4/6 inhibitors that act directly on the cell cycle, it is evident that the optimization of the combination with radiant treatments appears to be a goal to be achieved. In record studies of CDK4/6 inhibitors, radiotherapy is allowed before systemic therapies starting and is preferable to administer it not in concomitance[64]-[66]. Moreover, during systemic therapy indication to radiotherapy is usually for progressive disease, with shifting to another line. In literature, few data are reported that showed feasibility of radiotherapy in concomitance with CDK4/6 inhibitors, with a possible side effects arising (for example there are some reports of GI toxicity with RT on bone metastasis during abemaciclib)[67]-[70]. Another interesting issue about radiotherapy and CDK4/6 inhibitor is time for safety because these drugs cause a cell blockage in G1 phase with consequently radioresistance. At the end, hypotetic effect on immune system by CDK4/6 inhibitor could be implemented with ablative RT, and it is under investigation in phase II protocol ongoing.

PARP inhibitors and radiotherapy. For mBRCA metastatic breast cancer, some data are reported about safety and benefic effect of radiotherapy and PARP inhibitors, but it is possible that DNA damage is sinergically caused by combined effect of drug and radiotherapy.

HER2 target therapy and radiotherapy. Adjuvant studies on HER2+ target therapies such as trastuzumab, pertuzumab and lapatinib show that they are safely administered during radiotherapy[71]-[73]. Data about TDM-1 are few and not indicative of possible beneficial and side effects.

Immunotherapy and radiotherapy. Although immunotherapy has shown antitumor activity against several advanced tumors in recent years, in breast cancer only in TN disease showed promising results. In fact, atezolizumab plus nab-paclitaxel in PD-L1 positive metastatic TN population has showed an increase of PFS and OS respect chemotherapy alone[74]. The spread of bone metastases activates many immunosuppressive pathways. Therefore, the immunophenotype of bone metastases could represent a different pattern of 
response to immunotherapy when compared to visceral disease. Though checkpoint inhibitors have shown significant efficacy in many tumors including TN breast cancer with visceral metastases, their specific performance in bone metastases is not well understood and it may be poor. Although we currently have not clinical data, radiotherapy on bone metastases could make these localizations of disease more immunogenic and optimize the effectiveness of inhibitory checkpoints. Given these considerations, studying how and when to combine these treatments is an important goal of clinical research in the coming years.

Further perspectives. At the present time there is an increasing interest in oligometastatic breast cancer, especially in setting with good prognosis (Luminal subtype, single lesion, bone metastasis only). Ongoing trials are testing this setting of patients. On April 2020, a Medline on ClinicalTrial.gov showed that 6 trials were active for oligometastatic while only 1 trial was active for oligo progression.

A phase II trial (CLEAR, NCT03750396) is dedicated to oligometastatic recurrent patients (all parenchyma) with ER+/HER2- who underwent a radical local approach [surgery, radiotherapy ((57 97.5Gy/6 10 Fraction) or radiofrequency] during first systemic line to test PFS. Another trial (NCT02364557) is recruiting patients with limited MBC, randomizing them between systemic therapies (according to standard of care) and systemic therapies with association of stereotactic radiosurgery in 1,3 , or 5 fractions at the discretion of the treating physician, to test PFS and OS. A phase III study, STEREO-SEIN Trial, (NCT02089100) is testing the role of curative SBRT in de-novo oligometastatic breast cancer (no triple negative subtypes), randomizing patients between systemic therapies (according to standard of care) and systemic therapies with association of stereotactic radiosurgery. In another trial (NCT03808337), supported by Memorian Sloan Kettering Cancer Center, is recruiting metastatic non-small cell lung cancer or triple negative breast cancer, to be randomized between standard systemic therapies vs. receiving SBRT (with a minimum BED more than or equal to $48 \mathrm{~Gy}_{10}$ ) to all sites of metastasis, concurrently with systemic therapies. In another phase I/II trial by $\mathrm{NCl}$ (NCT00182793), patients with stage IV Metastatic and stage IIIB/C Breast Cancer were enrolled to 
receive bone marrow ablation with chemotherapy and autologous-autologous tandem hematopoietic stem cell transplantation and concurrent RT on site of disease. In this study, oligometastatic patients, received helical-tomotherapy RT on site of metastases with standard fractionation. In CIMER study (NCT04220476), a phase II study, patients with oligometastatic BC, luminal subtypes, who are candidates to first-line with CDK4/6 inhibitors will be randomizing between receiving first-line of treatment vs. underwent also immune-SBRT every $48 \mathrm{~h}$ on al sites of metastases with a total dose of $50 \mathrm{~Gy}$ in $5 \mathrm{Fr}$.

At the present time only 1 trial (NCT03808662) is testing oligoprogressive setting in NSCLS and TNBC patients, randomizing them between standard of care and SBRT 9-10 Gy $\times 3$ or 10 Gy $\times 5$ fractions given every other day to all oligoprogressive sites.

\section{Implication for clinicians}

According to time, disease presentation and prognosis, bone metastases from breast cancer patients can follow different pathway of care, for optimize symptoms management and outcomes.

In Figure 2, a pathway for palliative and radical setting are respectively reported.

\section{Conclusions}

Bone metastasis is a condition that unfortunately still affect patients with breast cancer, also limiting quality of life. Among these patients, oligometastatic breast cancer with only bone presentation represent a subgroup with favourable prognosis and in which escalation of diagnostic imaging methods, systemic therapies and imbrication with SBRT can be related with survival. Use of few or single fraction SBRT can allow physician to administered BED of $75 \mathrm{~Gy}$ and to treat, with a radical intent, patient who present good prognosis.

\section{Bibliography}


[1] F. Andre et al., «Breast Cancer With Synchronous Metastases: Trends in Survival During a 14-Year Period», JCO, vol. 22, n. 16, pagg. 3302-3308, ago. 2004, doi: 10.1200/JCO.2004.08.095.

[2] G. W. Sledge, «Curing Metastatic Breast Cancer», JOP, vol. 12, n. 1, pagg. 6-10, gen. 2016, doi: 10.1200/JOP.2015.008953.

[3] American Cancer Society, "Survival Rates for Breast Cancer». [In linea]. Available at: https://www.cancer.org/cancer/breast-cancer/understanding-a-breast-cancer-diagnosis/breastcancer-survival-rates.html.

[4] N. Harbeck et al., «Breast cancer», Nat Rev Dis Primers, vol. 5, n. 1, pag. 66, dic. 2019, doi: 10.1038/s41572-019-0111-2.

[5] R. K. Hernandez, S. W. Wade, A. Reich, M. Pirolli, A. Liede, e G. H. Lyman, «Incidence of bone metastases in patients with solid tumors: analysis of oncology electronic medical records in the United States», BMC Cancer, vol. 18, n. 1, pag. 44, dic. 2018, doi: 10.1186/s12885-017-3922-0.

[6] Q. Wu et al., «Breast cancer subtypes predict the preferential site of distant metastases: a SEER based study», Oncotarget, vol. 8, n. 17, apr. 2017, doi: 10.18632/oncotarget.15856.

[7] I. A. Molnár et al., "Breast carcinoma subtypes show different patterns of metastatic behavior», Virchows Arch, vol. 470, n. 3, pagg. 275-283, mar. 2017, doi: 10.1007/s00428-017-2065-7.

[8] M. Guckenberger et al., "Characterisation and classification of oligometastatic disease: a European Society for Radiotherapy and Oncology and European Organisation for Research and Treatment of Cancer consensus recommendation», The Lancet Oncology, vol. 21, n. 1, pagg. e18-e28, gen. 2020, doi: 10.1016/S1470-2045(19)30718-1.

[9] E. Eisenhauer et al., «New Response Evaluation Criteria in Solid Tumours: Revised RECIST Guideline (Version 1.1)», Eur J Cancer, vol. 45, n. 2, pagg. 228-47, 2009, doi: 10.1016/j.ejca.2008.10.026.

[10] G. Mundy, "Mechanisms of Bone Metastasis», Cancer, vol. 80, n. 8 Suppl, pagg. 1546-56, 1997, doi: 10.1002/(sici)1097-0142(19971015)80:8+<1546::aid-cncr4>3.3.co;2-r.

[11] S. D’Oronzo, R. Coleman, J. Brown, e F. Silvestris, «Metastatic bone disease: Pathogenesis and therapeutic options», Journal of Bone Oncology, vol. 15, pag. 100205, apr. 2019, doi: 10.1016/j.jbo.2018.10.004.

[12] L. A. M.-L. Vakaet e T. Boterberg, "Pain control by ionizing radiation of bone metastasis», Int. J. Dev. Biol., vol. 48, n. 5-6, pagg. 599-606, 2004, doi: 10.1387/ijdb.041817lv.

[13] S. Manfrida et al., "IMproved MAnagement (IM-MA study) in cancer-related pain: the value of a joint approach by an integrated team of radiotherapist and anesthetist», Support Care Cancer, vol. 27, n. 2, pagg. 505-512, feb. 2019, doi: 10.1007/s00520-018-4335-6.

[14] M. Cristofanilli et al., «Fulvestrant plus palbociclib versus fulvestrant plus placebo for treatment of hormone-receptor-positive, HER2-negative metastatic breast cancer that progressed on previous endocrine therapy (PALOMA-3): final analysis of the multicentre, double-blind, phase 3 randomised controlled trial», Lancet Oncol, vol. 17, n. 4, pagg. 425-439, apr. 2016, doi: https://doi.org/10.1016/S1470-2045(15)00613-0.

[15] P. Ghezzi, S. Magnanini, e M. Rinaldini, «Impact of Follow-up Testing on Survival and Health-Related Quality of Life in Breast Cancer Patients A Multicenter Randomized Controlled Trial», JAMA, vol. 271, $\mathrm{n}$. 20, pag. 1587, 1592, doi: 10.1001/jama.1994.03510440047031.

[16] M. Rosselli Del Turco, D. Palli, e A. Cariddi, «Intensive Diagnostic Follow-up After Treatment of Primary Breast Cancer. A Randomized Trial», JAMA, vol. 271, n. 20, pagg. 1593-1597, mag. 1994, doi: doi:10.1001/jama.1994.03510440053032.

[17] M. T. Milano, A. W. Katz, H. Zhang, C. F. Huggins, K. S. Aujla, e P. Okunieff, «Oligometastatic breast cancer treated with hypofractionated stereotactic radiotherapy: Some patients survive longer than a decade», Radiotherapy and Oncology, vol. 131, pagg. 45-51, feb. 2019, doi: 10.1016/j.radonc.2018.11.022.

[18] T. Kobayashi et al., "Possible clinical cure of metastatic breast cancer: lessons from our 30-year experience with oligometastatic breast cancer patients and literature review», Breast Cancer, vol. 19, n. 3, pagg. 218-237, lug. 2012, doi: 10.1007/s12282-012-0347-0. 
[19] G. Mundy, «Metastasis to bone: causes, consequences and therapeutic opportunities», Nature Rewiews Cancer, vol. 2, pagg. 584-593, 2002, doi: 10.1038/nrc867.

[20] E. Chow, J. Wu, P. Hoskin, L. Coia, S. Bentzen, e P. Blitzer, «International consensus on palliative radiotherapy endpoints for future clinical trials in bone metastases.", Radiotherapy and Oncology, vol. 64, n. 3, pagg. 275-80, 2002.

[21] E. Chow et al., "Update of the International Consensus on Palliative Radiotherapy Endpoints for Future Clinical Trials in Bone Metastases», International Journal of Radiation Oncology*Biology*Physics, vol. 82, n. 5, pagg. 1730-1737, apr. 2012, doi: 10.1016/j.ijrobp.2011.02.008.

[22] K. L. Zeng, C.-L. Tseng, H. Soliman, Y. Weiss, A. Sahgal, e S. Myrehaug, «Stereotactic Body Radiotherapy (SBRT) for Oligometastatic Spine Metastases: An Overview», Front. Oncol., vol. 9, pag. 337, mag. 2019, doi: 10.3389/fonc. 2019.00337.

[23] R. S. Schwartz e J. K. Erban, «Timing of Metastasis in Breast Cancer», N Engl J Med, vol. 376, n. 25, pagg. 2486-2488, giu. 2017, doi: 10.1056/NEJMcibr1701388.

[24] H. Hosseini et al., "Early dissemination seeds metastasis in breast cancer», Nature, vol. 540, pagg. 552558, 2016, doi: 10.1038/nature20785.

[25] K. Harper et al., "Mechanism of early dissemination and metastasis in Her2+ mammary cancer», Nature, vol. 540, pagg. 588-592, 2016, doi: 10.1038/nature20609.

[26] N. Ghanem et al., "Diagnostic value of MRI in comparison to scintigraphy, PET, MS-CT and PET/CT for the detection of metastases of bone», European Journal of Radiology, vol. 55, n. 1, pagg. 41-55, lug. 2005, doi: 10.1016/j.ejrad.2005.01.016.

[27] D. Di Gioia, P. Stieber, G. P. Schmidt, D. Nagel, V. Heinemann, e A. Baur-Melnyk, «Early detection of metastatic disease in asymptomatic breast cancer patients with whole-body imaging and defined tumour marker increase», Br J Cancer, vol. 112, n. 5, pagg. 809-818, mar. 2015, doi: 10.1038/bjc.2015.8.

[28] G. Cook e V. Goh, "Molecular imaging of bone metastases and their response to therapy", J Nucl Med, pag. jnumed.119.234260, apr. 2020, doi: 10.2967/jnumed.119.234260.

[29] S. Taralli, M. Lorusso, V. Scolozzi, V. Masiello, F. Marazzi, e M. L. Calcagni, «Response evaluation with 18F-FDG PET/CT in metastatic breast cancer patients treated with Palbociclib: first experience in clinical practice», Ann Nucl Med, vol. 33, n. 3, pagg. 193-200, mar. 2019, doi: 10.1007/s12149-018-01323-8.

[30] National Comprehensive Cancer Network, «NCCN Clinical Practice Guidelines in Oncology (NCCN Guidelines) Breast Cancer», vol. Version 2.2020, feb. 2020.

[31] S. L. Moore, M. J. Kransdorf, M. E. Schweitzer, M. D. Murphey, e J. S. Babb, «Can Sarcoidosis and Metastatic Bone Lesions Be Reliably Differentiated on Routine MRI?», American Journal of Roentgenology, vol. 198, n. 6, pagg. 1387-1393, giu. 2012, doi: 10.2214/AJR.11.7498.

[32] M. Soussan, A. Augier, P.-Y. Brillet, P. Weinmann, e D. Valeyre, «Functional Imaging in Extrapulmonary Sarcoidosis: FDG-PET/CT and MR Features», Clinical Nuclear Medicine, vol. 39, n. 2, pagg. e146-e159, feb. 2014, doi: 10.1097/RLU.0b013e318279f264.

[33] R. Foerster et al., "Histopathological Findings After Reirradiation Compared to First Irradiation of Spinal Bone Metastases With Stereotactic Body Radiotherapy: A Cohort Study", Neurosurgery, vol. 84, n. 2, pagg. 435-441, feb. 2019, doi: 10.1093/neuros/nyy059.

[34] F. Alongi, S. Arcangeli, A. R. Filippi, U. Ricardi, e M. Scorsetti, «Review and Uses of Stereotactic Body Radiation Therapy for Oligometastases», The Oncologist, vol. 17, n. 8, pagg. 1100-1107, ago. 2012, doi: 10.1634/theoncologist.2012-0092.

[35] S. Costa e M. R. Reagan, «Therapeutic Irradiation: Consequences for Bone and Bone Marrow Adipose Tissue», Front. Endocrinol., vol. 10, pag. 587, ago. 2019, doi: 10.3389/fendo.2019.00587.

[36] M. Goblirsch et al., "Radiation Treatment Decreases Bone Cancer Pain, Osteolysis and Tumor Size», Radiation Research, vol. 161, n. 2, pagg. 228-234, feb. 2004, doi: 10.1667/RR3108.

[37] J. Zhang et al., "Differences in responses to X-ray exposure between osteoclast and osteoblast cells», Journal of Radiation Research, vol. 58, n. 6, pagg. 791-802, nov. 2017, doi: 10.1093/jrr/rrx026.

[38] J. Steverink et al., "Early Tissue Effects of Stereotactic Body Radiation Therapy for Spinal Metastases», Int J Radiat Oncol Biol Phys, vol. 100, n. 5, pagg. 1254-1258, 2018, doi: 10.1016/j.ijrobp.2018.01.005. 
[39] R. Arriagada, H. Mouriesse, D. Sarrazin, R. Clark, e G. Deboer, «Analysis of tumor parameters, tumor dose and local control: the experience of the Gustave-Roussy Institute and the Princess Margaret Hospital», Int J Radiat Oncol Biol Phys, vol. 11, pagg. 1751-1757, 1985.

[40] P. C. Gerszten et al., "Single-fraction radiosurgery for the treatment of spinal breast metastases», Cancer, vol. 104, n. 10, pagg. 2244-2254, nov. 2005, doi: 10.1002/cncr.21467.

[41] D. Kwapisz, "Oligometastatic breast cancer», Breast Cancer, vol. 26, pagg. 138-146, 2019, doi: 10.1007/s12282-018-0921-1.

[42] E. Chow, K. Harris, G. Fan, M. Tsao, e W. M. Sze, «Palliative Radiotherapy Trials for Bone Metastases: A Systematic Review», JCO, vol. 25, n. 11, pagg. 1423-1436, apr. 2007, doi: 10.1200/JC0.2006.09.5281.

[43] D. Rades et al., "A prospective evaluation of two radiotherapy schedules with 10 versus 20 fractions for the treatment of metastatic spinal cord compression: Final results of a multicenter study», Cancer, vol. 101, n. 11, pagg. 2687-2692, dic. 2004, doi: 10.1002/cncr.20633.

[44] T. de Vin, B. Engels, T. Gevaert, G. Storme, e M. De Ridder, «Stereotactic radiotherapy for oligometastatic cancer: a prognostic model for survival», Annals of Oncology, vol. 25, n. 2, pagg. 467471, feb. 2014, doi: 10.1093/annonc/mdt537.

[45] A. C. Tree et al., «Stereotactic body radiotherapy for oligometastases», The Lancet Oncology, vol. 14, n. 1, pagg. e28-e37, gen. 2013, doi: 10.1016/S1470-2045(12)70510-7.

[46] F. Cellini et al., "Pain REduction with bone metastases STereotactic radiotherapy (PREST): A phase III randomized multicentric trial», Trials, vol. 20, n. 1, pag. 609, dic. 2019, doi: 10.1186/s13063-019-3676$\mathrm{X}$.

[47] M. Mizumoto et al., «Prognostic factors and a scoring system for survival after radiotherapy for metastases to the spinal column: A review of 544 patients at Shizuoka Cancer Center Hospital», Cancer, vol. 113, n. 10, pagg. 2816-2822, nov. 2008, doi: 10.1002/cncr.23888.

[48] A. C. Wong et al., "Clinical and molecular markers of long-term survival after oligometastasis-directed stereotactic body radiotherapy (SBRT): Survival After SBRT for Oligometastases», Cancer, vol. 122, n. 14, pagg. 2242-2250, lug. 2016, doi: 10.1002/cncr.30058.

[49] M. Trovo et al., "Radical radiation therapy for oligometastatic breast cancer: Results of a prospective phase II trial», Radiotherapy and Oncology, vol. 126, n. 1, pagg. 177-180, gen. 2018, doi: 10.1016/j.radonc.2017.08.032.

[50] G. S. Yoo, J. I. Yu, W. Park, S. J. Huh, e D. H. Choi, «Prognostic factors in breast cancer with extracranial oligometastases and the appropriate role of radiation therapy», Radiat Oncol J, vol. 33, n. 4, pag. 301, 2015, doi: 10.3857/roj.2015.33.4.301.

[51] T. Y. Kam, O. S. H. Chan, A. W. M. Hung, e R. M. W. Yeung, «Utilization of stereotactic ablative radiotherapy in oligometastatic \& oligoprogressive skeletal metastases: Results and pattern of failure», Asia-Pac J Clin Oncol, vol. 15, n. S2, pagg. 14-19, mar. 2019, doi: 10.1111/ajco.13115.

[52] A. Farooqi et al., «Outcomes After Hypofractionated Dose-Escalation using a Simultaneous Integrated Boost Technique for Treatment of Spine Metastases Not Amenable to Stereotactic Radiosurgery», Practical Radiation Oncology, vol. 9, n. 2, pagg. e142-e148, mar. 2019, doi: 10.1016/j.prro.2018.10.008.

[53] T. D. Nguyen et al., "Simultaneous high-dose external irradiation and daily cisplatin in unresectable, non-metastatic adenocarcinoma of the pancreas: a phase I-II study ", Radiotherapy and Oncology, vol. 45, n. 2, pagg. 129-132, nov. 1997, doi: 10.1016/S0167-8140(97)00116-3.

[54] M. Bonet et al., «Radiation Therapy for Bone-Only Metastases in Breast Cancer Patients: A GOCO Survey of Current Clinical Practice», Reports of Practical Oncology \& Radiotherapy, vol. 25, n. 1, pagg. 113-116, feb. 2020, doi: 10.1016/j.rpor.2019.12.019.

[55] F. Cardoso et al., "4th ESO-ESMO International Consensus Guidelines for Advanced Breast Cancer (ABC 4)", Annals of Oncology, vol. 29, n. 8, pagg. 1634-1657, ago. 2018, doi: 10.1093/annonc/mdy192.

[56] S. M. Radaideh e G. W. Sledge, «Taxane vs. taxane: is the duel at an end? A commentary on a phase-III trial of doxorubicin and docetaxel versus doxorubicin and paclitaxel in metastatic breast cancer: results of the ERASME 3 study», Breast Cancer Res Treat, vol. 111, n. 2, pagg. 203-208, set. 2008, doi: 10.1007/s10549-007-9776-4. 
[57] G. W. Sledge et al., «Phase III Trial of Doxorubicin, Paclitaxel, and the Combination of Doxorubicin and Paclitaxel as Front-Line Chemotherapy for Metastatic Breast Cancer: An Intergroup Trial (E1193)», JCO, vol. 21, n. 4, pagg. 588-592, feb. 2003, doi: 10.1200/JCO.2003.08.013.

[58] J. Cortes et al., «Eribulin monotherapy versus treatment of physician's choice in patients with metastatic breast cancer (EMBRACE): a phase 3 open-label randomised study", The Lancet, vol. 377, n. 9769, pagg. 914-923, mar. 2011, doi: 10.1016/S0140-6736(11)60070-6.

[59] W. J. Gradishar et al., «Significantly Longer Progression-Free Survival With nab -Paclitaxel Compared With Docetaxel As First-Line Therapy for Metastatic Breast Cancer», JCO, vol. 27, n. 22, pagg. 36113619, ago. 2009, doi: 10.1200/JCO.2008.18.5397.

[60] K. S. Albain, S. Nag, e G. Calderillo-Ruiz, «Global phase III study of gemcitabine plus paclitaxel (GT) vs. paclitaxel $(T)$ as frontline therapy for metastatic breast cancer $(\mathrm{MBC})$ : First report of overall survival [Abstract]», J Clin Oncol, vol. 22, n. Abstract 510, 2004.

[61] D. A. Yardley et al., «Phase II/III weekly nab-paclitaxel plus gemcitabine or carboplatin versus gemcitabine/carboplatin as first-line treatment of patients with metastatic triple-negative breast cancer (the tnAcity study): study protocol for a randomized controlled trial», Trials, vol. 16, n. 1, pag. 575, dic. 2015, doi: 10.1186/s13063-015-1101-7.

[62] M. Piccart et al., "Everolimus plus exemestane for hormone-receptor-positive, human epidermal growth factor receptor-2-negative advanced breast cancer: overall survival results from BOLERO-2», Annals of Oncology, vol. 25, n. 12, pagg. 2357-2362, dic. 2014, doi: 10.1093/annonc/mdu456.

[63] F. André et al., "Alpelisib for PIK3CA -Mutated, Hormone Receptor-Positive Advanced Breast Cancer», N Engl J Med, vol. 380, n. 20, pagg. 1929-1940, mag. 2019, doi: 10.1056/NEJMoa1813904.

[64] R. S. Finn et al., «Palbociclib and Letrozole in Advanced Breast Cancer», N Engl J Med, vol. 375, n. 20, pagg. 1925-1936, nov. 2016, doi: 10.1056/NEJMoa1607303.

[65] M. P. Goetz et al., "MONARCH 3: Abemaciclib As Initial Therapy for Advanced Breast Cancer», JCO, vol. 35, n. 32, pagg. 3638-3646, nov. 2017, doi: 10.1200/JCO.2017.75.6155.

[66] D. Tripathy et al., «Ribociclib plus endocrine therapy for premenopausal women with hormonereceptor-positive, advanced breast cancer (MONALEESA-7): a randomised phase 3 trial», The Lancet Oncology, vol. 19, n. 7, pagg. 904-915, lug. 2018, doi: 10.1016/S1470-2045(18)30292-4.

[67] N. B. Figura et al., «CDK 4/6 inhibitors and stereotactic radiation in the management of hormone receptor positive breast cancer brain metastases», J Neurooncol, vol. 144, n. 3, pagg. 583-589, set. 2019, doi: 10.1007/s11060-019-03260-6.

[68] J. A. Messer, E. Ekinci, T. A. Patel, e B. S. Teh, «Enhanced dermatologic toxicity following concurrent treatment with palbociclib and radiation therapy: A case report», Reports of Practical Oncology \& Radiotherapy, vol. 24, n. 3, pagg. 276-280, mag. 2019, doi: 10.1016/j.rpor.2019.03.001.

[69] M. Chowdhary et al., "Safety and Efficacy of Palbociclib and Radiation Therapy in Patients With Metastatic Breast Cancer: Initial Results of a Novel Combination», Advances in Radiation Oncology, vol. 4, n. 3, pagg. 453-457, lug. 2019, doi: 10.1016/j.adro.2019.03.011.

[70] E. Ippolito et al., «Concurrent radiotherapy with palbociclib or ribociclib for metastatic breast cancer patients: Preliminary assessment of toxicity», The Breast, vol. 46, pagg. 70-74, ago. 2019, doi: 10.1016/j.breast.2019.05.001.

[71] G. von Minckwitz et al., «Trastuzumab Emtansine for Residual Invasive HER2-Positive Breast Cancer», N Engl J Med, vol. 380, n. 7, pagg. 617-628, feb. 2019, doi: 10.1056/NEJMoa1814017.

[72] F. Mignot et al., "Concurrent administration of anti-HER2 therapy and radiotherapy: Systematic review», Radiotherapy and Oncology, vol. 124, n. 2, pagg. 190-199, ago. 2017, doi: 10.1016/j.radonc.2017.07.006.

[73] G. von Minckwitz et al., «Adjuvant Pertuzumab and Trastuzumab in Early HER2-Positive Breast Cancer», N Engl J Med, vol. 377, n. 2, pagg. 122-131, lug. 2017, doi: 10.1056/NEJMoa1703643.

[74] P. Schmid et al., "Atezolizumab and Nab-Paclitaxel in Advanced Triple-Negative Breast Cancer», N Engl J Med, vol. 379, n. 22, pagg. 2108-2121, nov. 2018, doi: 10.1056/NEJMoa1809615. 


\section{Table 1 - Radiotherapy Dose and Volumes for bone metastasis}

\begin{tabular}{|c|c|c|c|}
\hline Dose & Volume & Outcome & Reference \\
\hline $\begin{array}{l}8 \mathrm{~Gy} \\
1 \mathrm{Fr}\end{array}$ & $\begin{array}{l}\text { Bone compartment }+/ \text { - soft-tissue } \\
\text { invasion }\end{array}$ & $\begin{array}{l}\text { Symptom control (pain, neurological } \\
\text { compromission) } \\
\text { Preferable in case of poor } \\
\text { expectation of retreatments }\end{array}$ & $\begin{array}{l}\text { Chow E. 2002[20] } \\
\text { Chow E. 2007[42] } \\
\text { Chow E. 2012[21] }\end{array}$ \\
\hline $\begin{array}{l}20 \mathrm{~Gy} \\
5 \mathrm{Fr}\end{array}$ & $\begin{array}{l}\text { Bone compartment }+/ \text { - soft-tissue } \\
\text { invasion }\end{array}$ & $\begin{array}{l}\text { Symptom control (pain, neurological } \\
\text { compromission) }\end{array}$ & $\begin{array}{l}\text { Chow E. 2002[20] } \\
\text { Chow E. 2007[42] } \\
\text { Chow E. 2012[21] }\end{array}$ \\
\hline $\begin{array}{l}30 \mathrm{~Gy} \\
10 \mathrm{Fr}\end{array}$ & $\begin{array}{l}\text { Bone compartment }+/ \text { - soft-tissue } \\
\text { invasion }\end{array}$ & $\begin{array}{l}\text { Symptom control (pain, neurological } \\
\text { compromission) } \\
\text { After surgical stabilization }\end{array}$ & Rades D, 2004[43] \\
\hline \multicolumn{4}{|c|}{ Table $1 b$ - Dose and volumes for radical radiotherapy on bone metastasis } \\
\hline Dose & Volume & Outcome & Reference \\
\hline $\begin{array}{l}\text { EQD2 of } \\
57.3 \mathrm{~Gy} \\
{[38.3-} \\
70] \\
\text { BED } 60 \mathrm{~Gy} \\
\text { (obtained) }\end{array}$ & Bone lesion + margin (mm) & $\begin{array}{l}\text { 5-y OS } 83 \% \text { (BO vs. no-BO p 0.002) } \\
10-y \text { OS } 75 \% \text { (BO vs. no-BO p 0.002) } \\
\text { FFWM (BO vs. no-BO p 0.018) }\end{array}$ & Milano MT, 2019[17] \\
\hline $\begin{array}{l}\text { BED }>50 \\
\text { Gy }\end{array}$ & Bone lesion + margin $(\mathrm{mm})$ & $\begin{array}{l}\text { 3-y DPFS } 36.8 \% \\
5-y \text { LC } 66.1 \% \\
5-y \text { OS } 49 \% \\
\text { Univariate Analysis : } \\
\text { Higher RT dose }(p=0.002) \\
\text { Whole Lesion RT }(p=0.007)\end{array}$ & Yoo GS, 2015[50] \\
\hline $\begin{array}{l}30-45 \mathrm{~Gy} \\
3 \mathrm{Fr}\end{array}$ & Bone lesion + margin $(\mathrm{mm})$ & $\begin{array}{l}1-y \text { PFS } 75 \% \\
2-y \text { PFS } 53 \% \\
2-y \text { LC } 97 \% \\
2-y \text { OS } 95 \%\end{array}$ & Trovo M, 2017[49] \\
\hline $\begin{array}{l}15- \\
22.5 \mathrm{~Gy} \\
1 \mathrm{Fr}\end{array}$ & Bone lesion + margin $(\mathrm{mm})$ & 15-months pain control $96 \%$ & Gerszten PC, 2005[40] \\
\hline $\begin{array}{l}35 \mathrm{~Gy} \\
\text { (spinal) } \\
50 \mathrm{~Gy} \\
\text { (no } \\
\text { spinal) } \\
5 \mathrm{Fr}\end{array}$ & Bone lesion + margin $(\mathrm{mm})$ & $\begin{array}{l}\text { 1-y LC } 91.2 \% \\
\text { PFS } 10.1 \text { months } \\
\text { OS } 37.3 \text { months }\end{array}$ & Kam TY, 2019[51] \\
\hline
\end{tabular}




\begin{tabular}{|l|l|l|l|}
\hline $40 \mathrm{~Gy}$ & Bone lesion + margin (mm) & $1-\mathrm{y} \mathrm{LC} \mathrm{93 \%}$ & Farooqi A, 2018[52] \\
(GTV) & Whole Vertebra (WV) & OS 58\% & \\
$30 \mathrm{~Gy}$ & & & \\
(WV) & & & \\
$10 \mathrm{Fr}$ & & & \\
\hline
\end{tabular}

Table 2 - Ongoing Trials on Oligometastatic and Oligoprogressive Breast Cancer Patients

\begin{tabular}{|c|c|c|c|c|}
\hline Reference & Setting & Intervention & $\begin{array}{l}\text { Radiotherapy } \\
\text { Dose/Volumes }\end{array}$ & $\begin{array}{l}\text { Primary } \\
\text { Endpoints }\end{array}$ \\
\hline $\begin{array}{l}\text { CLEAR, Jeong J, } \\
\text { NCT03750396 }\end{array}$ & $\begin{array}{l}\text { Oligometastatic breast } \\
\text { cancer recurrence }(>12 \\
\text { months) } \\
\text { All site of metastases }\end{array}$ & $\begin{array}{l}\text { Surgery or Radiotherapy or } \\
\text { Radiofrequency on metastasis }\end{array}$ & $\begin{array}{l}\text { Total radiation dose } \\
\text { and fractions are } \\
\text { various according } \\
\text { to metastatic } \\
\text { lesions } \\
\text { (57 97.5Gy/6 10 } \\
\text { Fraction) }\end{array}$ & PFS \\
\hline $\begin{array}{l}\text { NRG Oncology, } \\
\text { NCT02364557 }\end{array}$ & Limited MBC & SBRT +/- Surgery & $\begin{array}{l}\text { Radiosurgery in } 1 \text {, } \\
3 \text {, or } 5 \text { fractions } \\
\text { (according to } \\
\text { discretion of } \\
\text { physician) }\end{array}$ & $\begin{array}{l}\text { PFS } \\
\text { OS }\end{array}$ \\
\hline $\begin{array}{l}\text { STEREO-SEIN, } \\
\text { NCT02089100 }\end{array}$ & $\begin{array}{l}\text { De-novo Oligometastatic } \\
\text { Breast Cancer, excluding } \\
\text { triple negative subtype }\end{array}$ & SBRT & $\begin{array}{l}\text { SBRT with radical } \\
\text { intent to all sites of } \\
\text { metastases }\end{array}$ & PFS \\
\hline $\begin{array}{l}\text { MSKCC, } \\
\text { NCT03808337 }\end{array}$ & $\begin{array}{l}\text { Metastatic NSCLC or } \\
\text { TNBC }\end{array}$ & $\begin{array}{l}\text { SBRT concurrently to systemic } \\
\text { therapy }\end{array}$ & $\begin{array}{l}\text { SBRT with a } \\
\text { minimum BED of } 48 \\
\text { Gy to all sites }\end{array}$ & PFS \\
\hline $\begin{array}{l}\mathrm{NCl}, \\
\mathrm{NCT00182793}\end{array}$ & Stage IIIb-IV BC & $\begin{array}{l}\text { RT on primary site or on site of } \\
\text { metastasis (oligometastatic), High- } \\
\text { dose chemotherapy, Autologous } \\
\text { Stem Cells Transplant }\end{array}$ & $\begin{array}{l}\text { Tomotherapy on } \\
\text { site of disease with } \\
\text { standard } \\
\text { fractionation }\end{array}$ & $\begin{array}{l}\text { 5-y Relapse- } \\
\text { Free-Survival } \\
\text { 5-y Overall- } \\
\text { Survival-Rate }\end{array}$ \\
\hline $\begin{array}{l}\text { CIMER, } \\
\text { NCT04220476 }\end{array}$ & $\begin{array}{l}\text { Oligometastatic, Luminal } \\
\text { BC }\end{array}$ & SBRT (Immune-SBRT every $48 \mathrm{~h}$ ) & $\begin{array}{l}\text { SBRT every } 48 \mathrm{~h} \text {, to } \\
\text { all sites of } \\
\text { metastases } \\
50 \mathrm{GY} \text { in } 5 \text { fractions }\end{array}$ & $\begin{array}{l}\text { ORR } \\
\text { PFS } \\
\text { OS }\end{array}$ \\
\hline $\begin{array}{l}\text { MSKCC } \\
\text { NCT03808662 }\end{array}$ & $\begin{array}{l}\text { Oligoprogressive NSCLC } \\
\text { or TNBC }\end{array}$ & SBRT & $\begin{array}{l}\text { SBRT 9-10 Gy } \times 3 \text { or } \\
10 \text { Gy } \times 5 \text { fractions } \\
\text { given every other } \\
\text { day to all sites }\end{array}$ & PFS \\
\hline
\end{tabular}


Hypofractionation or Stereotactic Radiation Therapy (SBRT)

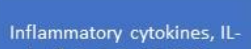

$1 \alpha / \beta, L-6,1 L-17, T N F-\alpha$

VEGF and senescence-

associated secretory
phenotype (SASP) proteins
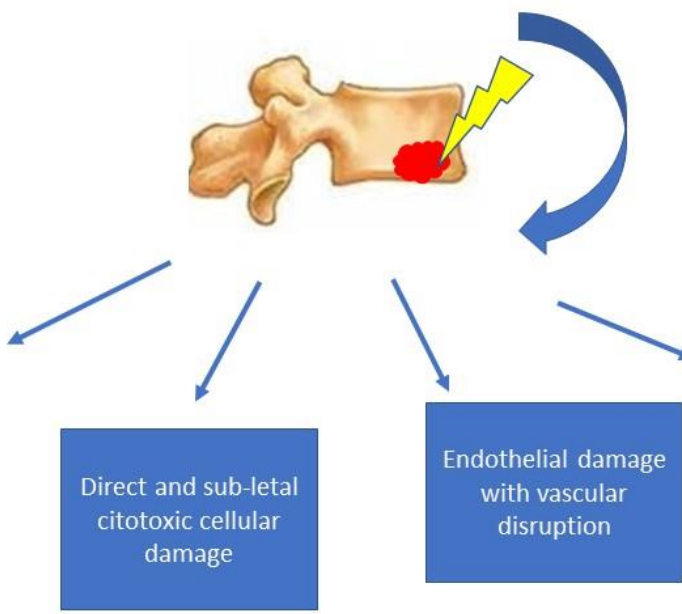

Long term

osteoclasts

depletion

Figure 1 - Radiotherapy Tissue Damage Mechanisms in Bone Metastases 


\section{GOOD PROGNOSIS BONE METASTATIC BREAST CANCER}

- De-novo oligometastatic bone-only breast cancer: breast cancer with less than 5 bone metastases at diagnosis

- Recurrent oligometastatic bone-only breast cancer: breast cancer with less than 5 bone metastases at recurrence

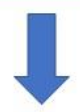

Evaluate enrollment in protocols

Evaluate SRS/SBRT to all sites of metastases (BED>75 Gy)

Evaluate SRS/SBRT in case of oligoprogression

Concomitance with drugs only if allowed by guidelines or in protocols

\section{INTERMEDIATE PROGNOSIS BONE METASTATIC BREAST CANCER}

- De-novo plurimetastatic bone-only breast cancer: breast cancer with more than 5 bone metastases at diagnosis

- De-novo oligometastatic bone-parenchimal breast cancer: breast cancer with less than 5 bone metastases at diagnosis

- Recurrent plurimetastatic bone-only breast cancer: breast cancer with more than 5 bone metastases at recurrence

- Recurrent oligometastatic bone-parenchimal breast cancer: breast cancer with less than 5 bone metastases at recurrence

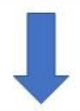

Systemic therapies priority

Evaluate RT (also SBRT) for symptomatic or critic sites

Evaluate SRS/SBRT in case of oligoprogression

Concomitance with drugs only if allowed by guidelines or in protocols 


\section{POOR PROGNOSIS BONE METASTATIC BREAST CANCER}

- De-novo plurimetastatic bone-parenchimal breast cancer: breast cancer with more than 5 bone metastases at diagnosis

- Recurrent plurimetastatic bone-parenchimal breast cancer: breast cancer with more than 5 bone metastases at recurrence

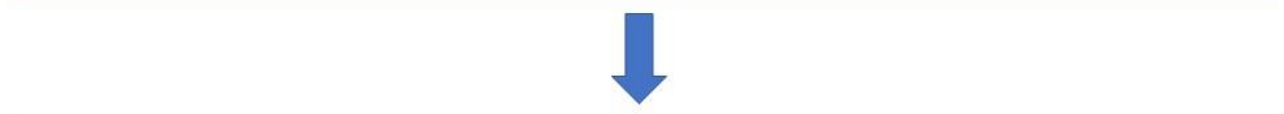

Systemic therapies, if indicated

Evaluate RT for symptomatic or critic sites

Modulate RT dose and fractions according to prognosis

Evaluate SBRT in case of re-treatment

Figure 2 - Therapeutic algorithms approach to patients with Bone metastases from breast cancer according to good, intermediate or poor prognosis. 